\title{
(2) OPEN ACCESS \\ Development of a core outcome set for lateral elbow tendinopathy (COS-LET) using best available evidence and an international consensus process
}

\author{
Marcus Bateman (D) ,' Jonathan P Evans (D) , ${ }^{2,3}$ Viana Vuvan, ${ }^{4}$ Val Jones, ${ }^{5}$ \\ Adam C Watts, ${ }^{6}$ Joideep Phadnis, ${ }^{7,8}$ Leanne M Bisset, ${ }^{9,10}$ Bill Vicenzino (1) , ${ }^{4}$ COS-LET \\ Authorship Group
}

\begin{abstract}
- Additional supplemental material is published online only. To view, please visit the journal online (http://dx.doi. org/10.1136/bjsports-2021 105044)
\end{abstract}

For numbered affiliations see end of article.

Correspondence to Marcus Bateman, Orthopaedic Department, University Hospitals of Derby and Burton NHS Foundation Trust, Derby, UK; marcus.bateman@nhs.net

$M B$ and JPE are joint first authors.

Accepted 14 January 2022

Check for updates

(c) Author(s) (or their employer(s)) 2022. Re-use permitted under CC BY. Published by BMJ.

To cite: Bateman $\mathrm{M}_{\text {, }}$ Evans JP, Vuvan $\mathrm{V}$, et al Br J Sports Med Epub ahead of print: [please include Day Month Year]. doi:10.1136/ bisports-2021-105044

\begin{abstract}
Objectives To develop a core outcome set for lateral elbow tendinopathy (COS-LET) and to provide guidance for outcome evaluation in future studies.

Methods We implemented a multi-stage mixedmethods design combining two systematic reviews, domain mapping of outcome measurement instruments to the core domains of tendinopathy, psychometric analysis of instruments, two patient focus groups and a Delphi study incorporating two surveys and an international consensus meeting. Following the OMERACT guidelines, we used a 70\% threshold for consensus.
\end{abstract}

Results 38 clinicians/researchers and 9 patients participated. 60 instruments were assessed for inclusion. The only instrument that was recommended for the COSLET was Patient Rated Tennis Elbow Evaluation (PRTEE) for the disability domain. Interim recommendations were made to use: the PRTEE function subscale for the function domain; PRTEE pain subscale items 1, 4 and 5 for the pain over a specified time domain; pain-free grip strength for the physical function capacity domain; a Numerical Rating Scale measuring pain on gripping for the pain on activity/loading domain; and time off work for the participation in life activities domain. No recommendations could be made for the quality-of-life, patient rating of condition and psychological factors domains.

Conclusions The COS-LET comprises the PRTEE for the disability domain. Interim-use recommendations included PRTEE subscales, time off work, pain-free grip strength and a Numerical Rating Scale measuring pain on gripping. Further work is required to validate these interim measures and develop suitable measures to capture the other domains.

\section{INTRODUCTION}

Background and objectives

Pain arising from the tendons on the lateral side of the elbow is common in adults, particularly in middle age. ${ }^{1}$ Historically, it has been known by various names such as lateral epicondylitis or tennis elbow, but the current accepted description is lateral elbow tendinopathy (LET). ${ }^{2}$ It is acknowledged that there is substantial heterogeneity of outcome measure instrument use in elbow research and specifically for LET. $^{3}$ With no clear consensus on which instruments most accurately represent a patient's LET-related health status, comparison of effectiveness research and evidence synthesis/metaanalysis has been hampered.

In 2019, an international group of experts in the field of tendinopathy (International Scientific Tendinopathy Symposium Consensus (ICON) Group), comprising researchers, healthcare professionals and patients, published a consensus document defining the nine health-related core domains of tendinopathy. That group recommended researchers and clinicians measure outcomes for specific regional tendinopathies against these domains. ${ }^{4}$

The aim of this project was to develop a core outcome set (COS) for LET that maps to the nine domains. A COS is a minimal set of outcome measures to be used in future research and clinical practice involving people with LET. It enables metaanalysis of findings from different studies using a consistent set of measures. To be included in a COS, measures need to be both practical to perform (based on cost, patient burden and availability) and of high quality (valid, responsive, reliable, interpretable and of acceptable burden for patients and investigators). ${ }^{5}$ The result will be a minimum set of outcome measurement instruments to be used in future LET research that allows direct comparison between different studies across the nine domains.

\section{Scope}

This COS relates to all adults diagnosed with LET and applies to interventional research (including surgical and non-surgical) and longitudinal assessment. The COS will only apply to the English language.

\section{METHOD}

We designed the project following the COSMINCOMET guideline. ${ }^{6}$ We developed a COS that was based on a consensus of perspectives gained from healthcare professionals with expertise in LET and patients with the condition. This involved a multistage stepwise process, which started with identifying the instruments used in studies of LET by updating a previous systematic evaluation of patientrated outcomes for LET. $^{7}$ These instruments were then mapped by the steering committee to the nine core tendinopathy domains. ${ }^{4}$ The mapped outcome measurement instruments were then subjected to the OMERACT truth (part a) and feasibility filters ${ }^{8}$ by participants in the first round of a Delphi survey. We (MB and JPE) then systematically evaluated the 
psychometric properties of the included instruments-applying the OMERACT truth (part b) and discrimination filters, ${ }^{8}$ using the EMPRO tool. ${ }^{9}$ This information then formed the basis of the second Delphi survey, which was conducted to make recommendations for a COS-LET. Focus groups were conducted with patients to review findings after Delphi survey 2. The results of the surveys and focus groups were then reviewed and discussed by participants at an international consensus online meeting (Delphi stage 3), before voting to determine the final COS-LET. The study was led by an international eight-person steering committee with expertise in LET-comprising a mix of junior and senior researchers and clinicians from surgical and physiotherapy backgrounds.

\section{Protocol/ registry entry}

We registered the project with the Core Outcome Measures in Effectiveness Trials Initiative (http://www.comet-initiative.org/ Studies/Details/1497) and published the protocol in an open access journal. (https://trialsjournal.biomedcentral.com/articles/ 10.1186/s13063-021-05291-9). This report follows the COS Standards for Reporting checklist. ${ }^{10}$

\section{Participants}

The Delphi study population comprised of experienced clinicians and researchers nominated by the steering committee, identified by their reputation as elbow clinical specialists or prior publications related to LET. Additionally, a search of the Expertscape ${ }^{11}$ and SCOPUS ${ }^{12}$ databases by author and filtered by the terms 'tennis elbow' and 'trial' identified a list of other researchers to approach. Representation from a range of nationalities, with a spread of ethnicity and sex was ensured.

Patient representatives were invited by the clinicians on the steering committee.
Information sources

In order to comprehensively evaluate all outcome measurement instruments used in research of LET, we systematically reviewed the literature. To do this, we updated the 2019, Evans et $a l^{7}$ systematic review of English language instruments used in surgical and non-surgical trials for LET (census date: 1 May 2017). The search results were screened initially by title and abstract by two reviewers (MB and JPE) independently of each other using the online Rayyan tool ${ }^{13}$ — any disagreements were discussed and reconciled. We included all study designs except research protocols, case studies and small case series of less than five patients. One hundred and ninety nine full texts from the original search and 93 from the updated searches (to February 2020) were screened down to 256 papers for data extractionproviding a comprehensive list of instruments used in LET research (figure 1). Extracted data included all outcome instruments used, number of patients included in the study and full details of any novel instruments.

\section{Consensus process and outcome scoring}

The retrieved instruments were then submitted to a stepwise consensus process that mapped them to the core tendinopathy domains. The mapped instruments where then used to construct the first survey. The instruments agreed to in that survey were then evaluated for their psychometrics-the results of which were included in a second survey. Results of the surveys were discussed in two patient focus groups. Finally, a consensus meeting reviewed and discussed findings before voting on the final COS-LET.

Instruments mapped to domains: the steering committee members mapped each instrument to the nine core tendinopathy domains ${ }^{4}$ : patient rating of condition; participation in life activities (day to day, work and sport); pain on activity/loading;

\section{Raw data from Evans et al (2019) systematic review: \\ Studies related to LET up to $2017(n=199)$}

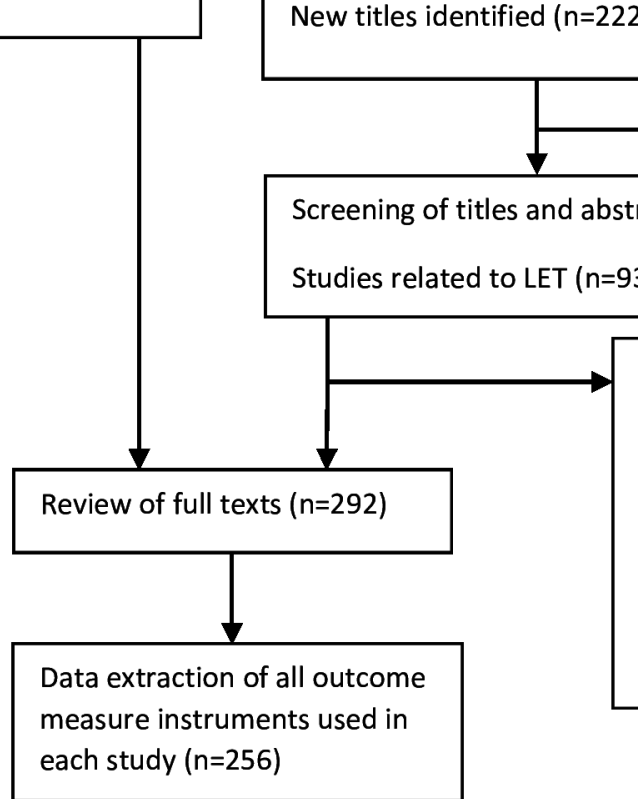

Search strategy used by Evans et al (2019) repeated with filter from 2017 onwards:

New titles identified $(n=2224)$ Non-English full text $(n=14)$ Medial Elbow Tendinopathy population $(n=5)$ Validation paper $(n=5)$ Duplicate from $2017(n=5)$ Protocol $(n=4)$ News article/commentary $(n=2)$ No full text available $(n=1)$

Figure 1 Adapted PRISMA flowchart: to review the outcome measure instruments used in all LET studies. LET, lateral elbow tendinopathy; PRISMA, Preferred Reporting Items for Systematic Reviews and Meta-Analyses. 


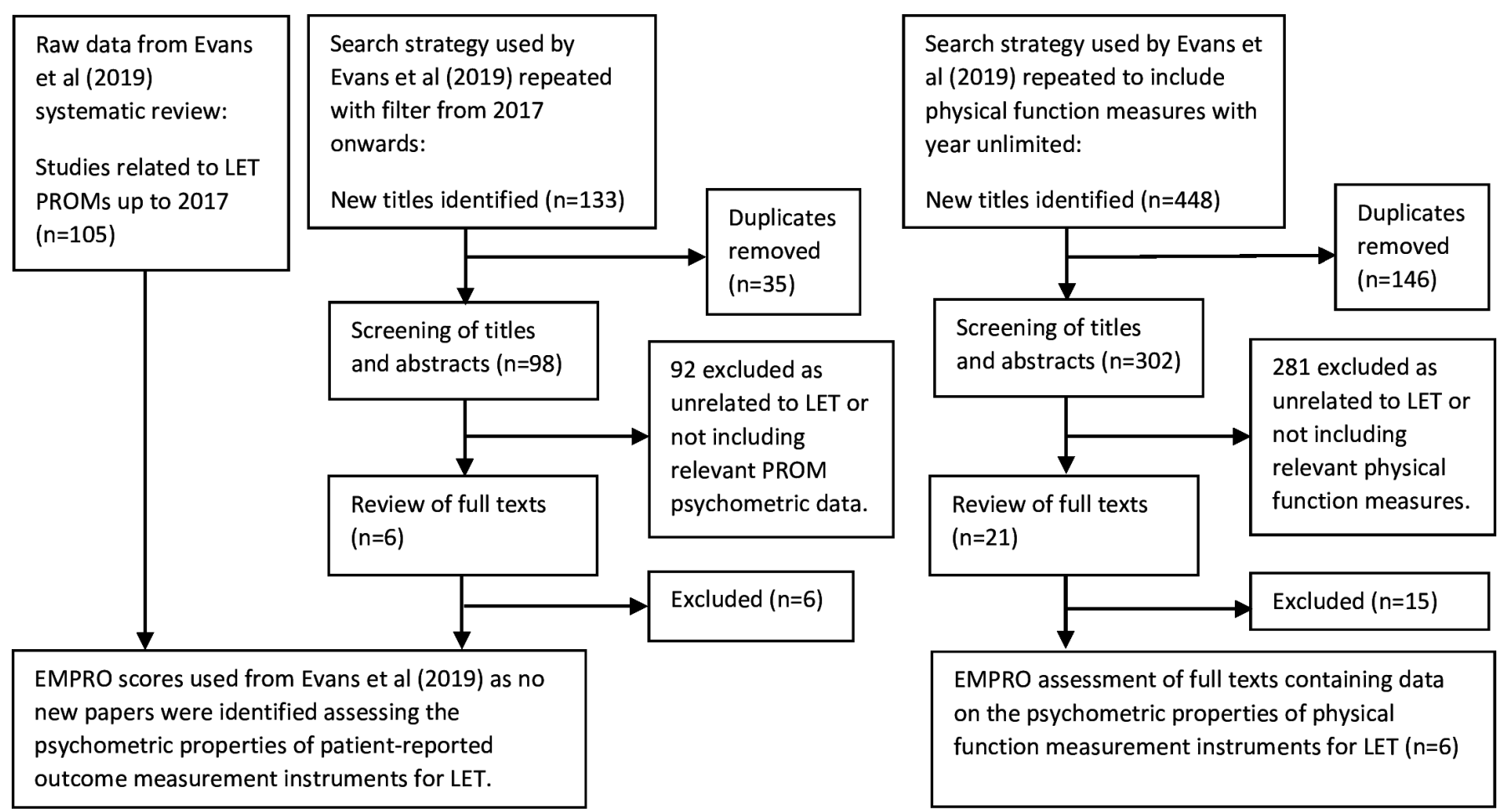

Figure 2 Adapted PRISMA flowchart: to review the psychometric properties of the instruments included after Delphi round 1. LET, lateral elbow tendinopathy; PRISMA, Preferred Reporting Items for Systematic Reviews and Meta-Analyses; PROMs, Patient-Reported Outcome Measures.

function; psychological factors; physical function capacity; disability; quality of life; and pain over a specified time. Each instrument was mapped by two steering committee members independently, then compared and reconciled if needed-using the instrument's published development study or manual.

Survey 1: each instrument and its reference document, including original development study and/or manual, were presented per mapped domain to participants in the first online survey using Qualtrics (Provo, Utah, USA). Participants were asked to respond yes/no/unsure whether each instrument was a truthful measure of the domain (valid), feasible to use clinically and whether it should be included in the COS-LET.

Outcomes were scored using the OMERACT traffic light system, ${ }^{8}$ whereby responses achieving $<30 \%$ agreement were rated red and excluded; those achieving $\geq 70 \%$ were rated green and included; and those achieving 30\%-69\% were rated amber, inconclusive but not excluded.

Psychometric evaluation of instruments: following exclusion of instruments ( $<30 \%$ agreement in survey 1$)$ and inclusion of any new instruments proposed by respondents, the OMERACT truth (part b) and discrimination filters were applied by two members of the steering committee (MB and JPE). This involved an update of Evans et al's systematic review to identify instrument development or validation studies (figure 2). These members used the EMPRO tool, separately to each other, to assess the psychometric properties (construct validity, reliability, repeatability, responsiveness and interpretability) of each instrument, then meeting to discuss points of contention. ${ }^{9}$ These were resolved without the need of a third assessor. The steering committee then voted anonymously, using the OMERACT traffic light system, on whether each instrument should be considered 'Good to go', 'A concern/more work needed' or 'Stop, do not continue'. 8 This voting stage was included to ratify the psychometric evaluation process and was guided by an EMPRO score threshold of $40 \%$ for inclusion.

Survey 2: during the second Delphi survey, participants were presented with the findings of Delphi survey 1 (online supplemental file 1), showing the traffic light rating of each instrument within their associated matched domain, and subsequent outcome of the truth (part b) and discrimination filters (online supplemental file 2). Participants were asked to rate instruments that achieved a nominal EMPRO score of $\geq 40 \%$ for inclusion in the final COS-LET (yes/no/unsure). Those instruments that were no or unsure for the final COS-LET, had no psychometric data or had an EMPRO score of $<40 \%$ were rated for interim use (yes/no). The responses were analysed and those instruments achieving $<30 \%$ of votes were excluded.

Patient focus groups: results of the Delphi stages, inclusive of survey 2, were then discussed at an online patient focus group for UK patients and another for Australian patients. Patients were asked to provide their insights/perspectives on the decisions to date and to ratify any instruments voted $\geq 70 \%$.

Final consensus meeting: participants attended an online consensus meeting to discuss the findings of the Delphi process to date (including patient focus group outcomes) and to vote on outcome measures in the COS-LET and for interim use. A report of the results of previous surveys and patient focus groups (online supplemental file 3) was provided to the participants 2 weeks prior.

\section{Consensus definition}

For each domain, instruments voted for by $\geq 70 \%$ of participants in both surveys and at the meeting were included in the COSLET. For domains where no instruments were agreed, interim suggestions were proposed based on a green light from Delphi 
Table 1 Participant characteristics (n (\%) unless otherwise stated)

\begin{tabular}{|c|c|c|c|c|}
\hline \multirow[b]{2}{*}{ Characteristics } & \multicolumn{3}{|c|}{ Clinicians/researchers } & \multirow{2}{*}{$\begin{array}{l}\text { Patients } \\
\begin{array}{l}\text { Survey } 1 / \text { focus group } \\
(n=9)\end{array}\end{array}$} \\
\hline & $\begin{array}{l}\text { Survey } 1 \\
(n=37)\end{array}$ & $\begin{array}{l}\text { Survey 2* } \\
(\mathrm{n}=37)\end{array}$ & Meeting $(n=31)$ & \\
\hline Sex: male & $25(67.6)$ & $25(67.6)$ & $22(71.0)$ & $4(44.4)$ \\
\hline Age: median (IQR; minimum-maximum), years & $51(43-57 ; 34-68)$ & $51(43-55 ; 34-68)$ & $51(43-53 ; 34-68)$ & $48(37-53 ; 26-59)$ \\
\hline \multicolumn{5}{|l|}{ Role } \\
\hline Clinician & $2(5.4)$ & $2(5.4)$ & $2(6.4)$ & NA \\
\hline Researcher & $5(13.5)$ & $5(13.5)$ & $4(12.9)$ & NA \\
\hline Clinician researcher & $30(81.1)$ & $30(81.1)$ & $25(80.7)$ & NA \\
\hline \multicolumn{5}{|l|}{ Highest academic qualification } \\
\hline $\mathrm{PhD}$ & $21(56.8)$ & $21(56.8)$ & $17(54.8)$ & - \\
\hline Master & $6(16.2)$ & $6(16.2)$ & $5(16.1)$ & $2(22.2)$ \\
\hline Doctor of Medicine & $7(18.9)$ & $7(18.9)$ & $7(22.6)$ & - \\
\hline Postgraduate diploma/certificate & - & - & - & $2(22.2)$ \\
\hline Bachelor & $3(8.1)$ & $3(8.1)$ & $2(6.5)$ & $4(44.4)$ \\
\hline No university qualification & - & - & - & $1(11.1)$ \\
\hline \multicolumn{5}{|l|}{ Profession } \\
\hline Physiotherapy & $16(43.2)$ & $16(43.2)$ & $14(45.2)$ & NA \\
\hline Orthopaedic surgeon & $14(37.8)$ & $14(37.8)$ & $12(38.7)$ & NA \\
\hline Sports and exercise medicine physician & $3(8.1)$ & $3(8.1)$ & $2(6.4)$ & NA \\
\hline Not specified & $3(8.1)$ & $3(8.1)$ & $2(6.4)$ & NA \\
\hline Rheumatologist & $1(2.7)$ & $1(2.7)$ & $1(3.2)$ & NA \\
\hline Therapy radiographer & - & - & - & $1(11.1)$ \\
\hline Health information technology & - & - & - & $1(11.1)$ \\
\hline Non-healthcare professional & - & - & - & $7(77.8)$ \\
\hline \multicolumn{5}{|l|}{ Lateral elbow tendinopathy } \\
\hline Current case & $1(2.7)$ & $1(2.7)$ & $1(3.2)$ & $5(55.6)$ \\
\hline History & $10(27.0)$ & $11(29.7)$ & $9(29.0)$ & $6(66.7)$ \\
\hline \multicolumn{5}{|l|}{ Country where work } \\
\hline Europe & $20(54.1)$ & $20(54.1)$ & $16(51.2)$ & $5(55.6)$ \\
\hline Australia & $11(29.7)$ & $10(27.0)$ & $8(25.8)$ & $4(44.4)$ \\
\hline North America & $5(13.5)$ & $5(13.5)$ & $5(16.1)$ & - \\
\hline Asia & $1(2.7)$ & $2(5.4)$ & $2(6.5)$ & - \\
\hline
\end{tabular}

*1 person from Australia did survey 1 but not 2; another did survey 2 not 1 (technical issues).

†Countries grouped per continent as follows: Europe=Belgium, Finland, Greece, Italy, the Netherlands, Norway, Spain, Sweden, Turkey (Istanbul) and UK; North America=Canada and USA; and Asia=India and Israel.

survey $1(\geq 70 \%)$, and amber light from Delphi survey 2 (30\%$69 \%$ ) and $\geq 70 \%$ agreement from the consensus meeting vote.

\section{RESULTS}

We commenced this study in January 2020, with regular steering committee working meetings to plan and design data collection. Data collection was completed at the consensus meeting on 5 May 2021.

\section{Protocol deviations}

The only deviation from the published protocol was that patient focus groups were conducted, rather than one-to-one interviews. This decision was taken to allow for patient interaction and group discussion, the impact of which on our findings is likely low to negligible.

\section{Participants}

We invited 58 healthcare professionals of which 40 agreed to participate, 7 did not agree (retired (2) and no reason given (5)) and 11 did not respond (maternity leave out of office message (1) and unknown reason (10)). Thirty eight engaged with the process and 2 withdrew. Thirty six (90\%) of the clinicians/ researchers who agreed to participate fully completed both surveys (table 1), 2 (5\%) completed one survey and 31 (84\%) of those completing surveys attended the online meeting. The clinician/researcher cohort consisted mainly of physiotherapists or orthopaedic surgeons, located in Europe or Australia, and had research higher degree training.

Nine patients from the UK and Australia participated in the study -7 completed the first survey and 5 participated in the focus groups.

\section{Outcomes}

Sixty unique instruments were identified from the first systematic review and included in survey 1 (table 2). From survey 1, three measures: pain on gripping, Patient Rated Tennis Elbow Evaluation (PRTEE) ${ }^{14-16}$ and Quick Disability of Arm, Shoulder and Hand Questionnaire (DASH), ${ }^{17}$ were the only ones reaching $\geq 70 \%$ for both the patient and the clinician/researcher groups. The Patient Specific Function Scale ${ }^{18}$ (function domain) and Tennis Elbow Functional Scale ${ }^{19}$ (both pain domains) were voted to be in the COS-LET by patients who commented favourably on the scores' item level face validity-however, the lack of robust psychometric evaluation in LET populations precluded 


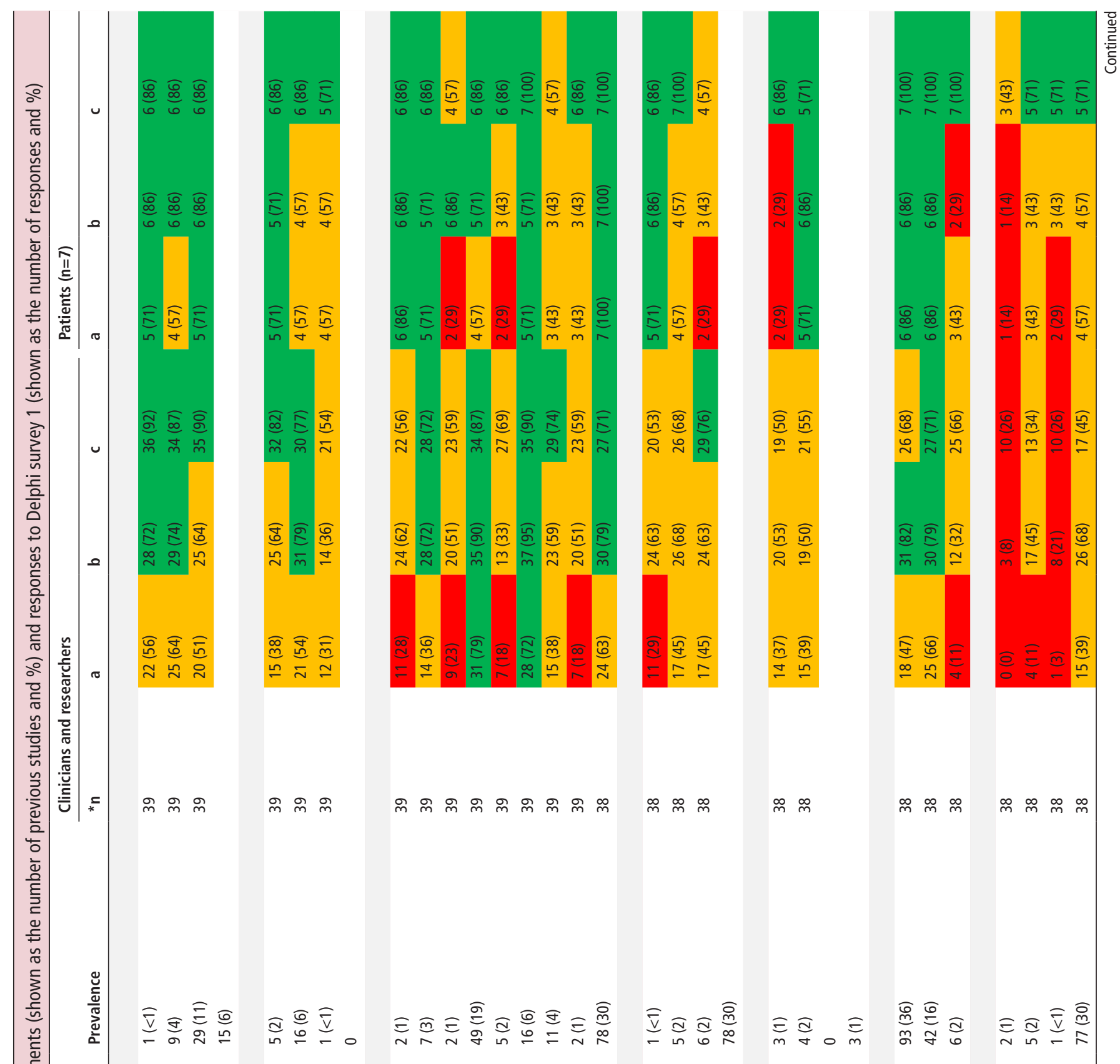

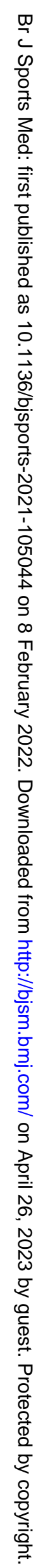




\section{Original research}

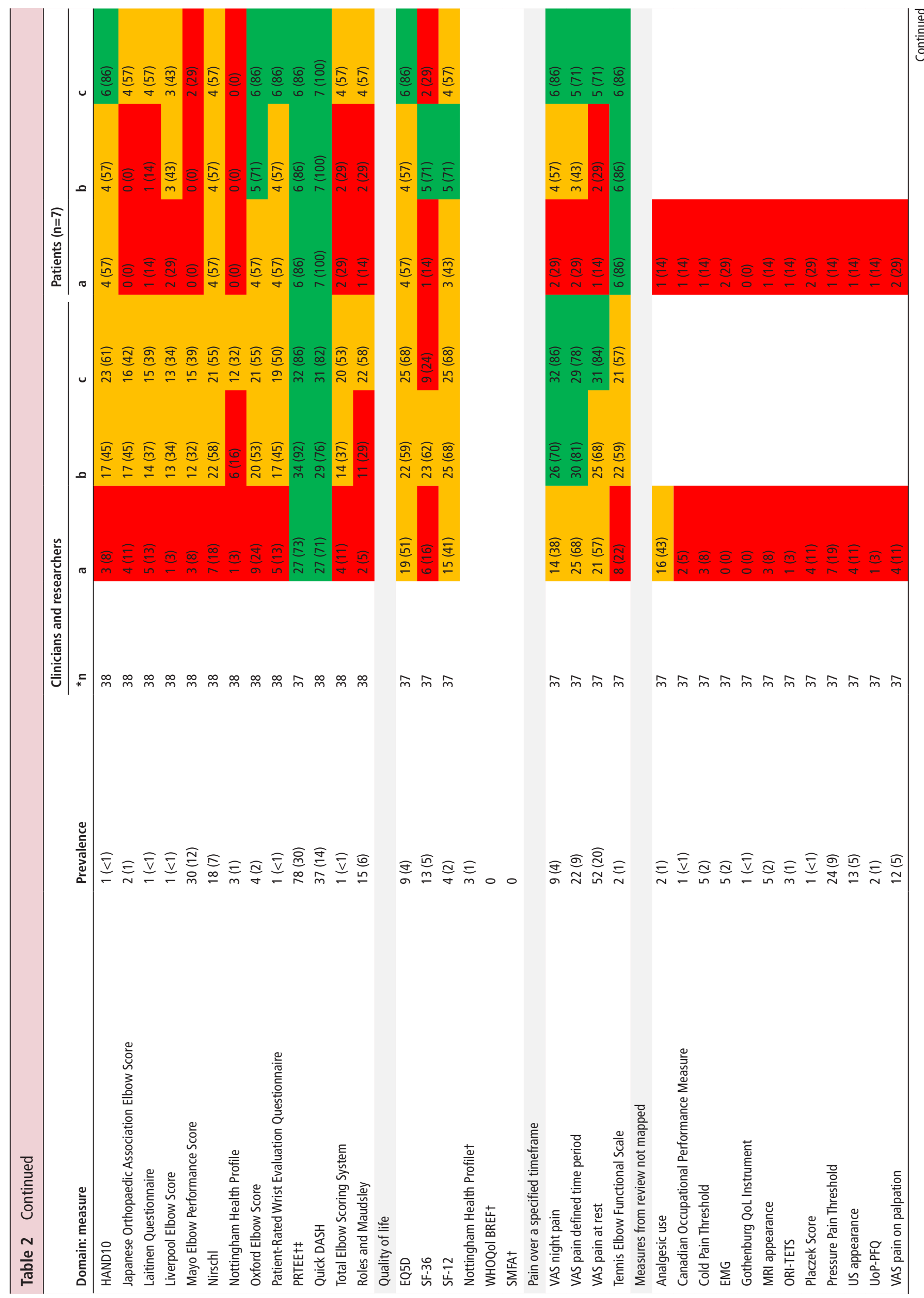




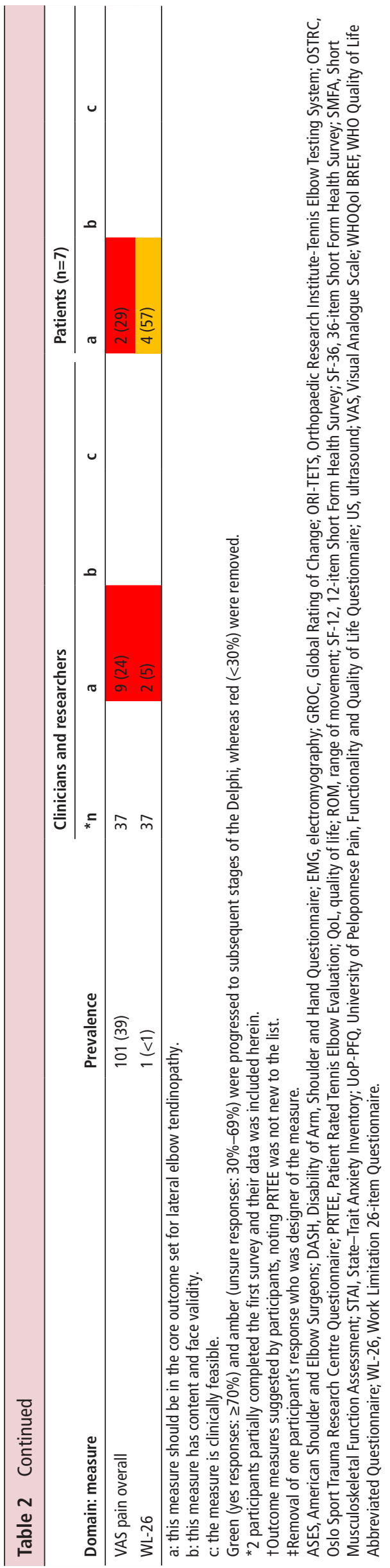

their inclusion following clinician/researcher evaluation (table 2). Twenty four instruments were excluded after this survey as they received $<30 \%$ of votes for inclusion in the COS-LET by both patients and clinicians/researchers. Participants proposed an additional seven instruments (table 2).

\section{Core outcome set}

A search for studies of the measures with $\geq 30 \%$ responses from survey 1 revealed that only 8/21 (38\%) instruments had been evaluated for their psychometric properties in specific LET populations. These measures were submitted to analysis with the EMPRO tool-scoring between 25\% and 73\% (table 3). The additional instruments proposed by responders in survey 1 had no psychometric data for the LET population and were not considered further in the development of the COS-LET. Seven instruments had an EMPRO score of $\geq 40$ and received $\geq 70 \%$ of votes in survey 1 from either patients or clinicians/researchersthey were: DASH, ${ }^{17}$ 20-22 Quick DASH, ${ }^{17} 2324$ Oxford Elbow Score, ${ }^{25}{ }^{26}$ PRTEE, ${ }^{14-16}$ Tennis Elbow Functional Scale, ${ }^{19}$ as well as grip strength. ${ }^{27-29}$ These were then independently assessed by the steering committee using the OMERACT truth (part b) and discrimination filters (results given in online supplemental file 2), which along with results from survey 1 , were available to inform clinician's/researcher's decisions in survey 2. The results of survey 2 are presented in table 3-only the PRTEE met the threshold for inclusion in the COS-LET for the disability domain, which was ratified at the consensus meeting.

\section{Interim recommendations}

Where there was no measure in a domain that met the criteria for the COS-LET, we considered measures that reached $\geq 70 \%$ hurdle in survey 1 -aiming to recommend one per domain to be used in the interim and as a direction for future research (table 3, Part B).

Of the measures that had psychometric data-that is, Tennis Elbow Functional Scale, maximum grip strength, pain-free grip strength and the PRTEE pain and function subscales-only the latter was voted as an interim measure in survey 2 (table 3 ). The patients (focus groups) agreed that items/subscales of the PRTEE (for pain on loading/activity, function and pain over specified time domains) and pain-free grip strength (for physical function capacity domain) were relevant to their condition. The consensus meeting decided that relevant subscale items from the PRTEE would be recommended as interim measures for function and pain over specified time domains. Pain-free grip strength was selected over maximum grip strength as it was thought to be more clinically and patient relevant. The meeting decided that pain on gripping, which had support in survey 1 but with no psychometric data, would be the preferred measure for pain on loading/activity domain, instead of the relevant subscale items from the PRTEE (table 3).

Of the other measures that did not have psychometric data (see table 3), only time off work was voted as an interim measure in survey 2 -it was ratified at the consensus meeting.

Notably, after the consensus meeting, there were three domains for which no interim measures were agreed. These were: quality of life, participant overall rating of condition and psychological.

\section{DISCUSSION}

This is the first attempt to determine the minimum COS-LET. We reached agreement for an outcome measure for one of the nine tendinopathy domains-PRTEE for disability. Although the PRTEE has been found to be psychometrically robust, we 
Table 3 Results of the clinimetric evaluation (EMPRO), second survey, patient focus group and final consensus meeting decision arranged in reverse chronology across the table-for each measure per domain. Data are frequency count (\%) unless otherwise specified. The COS-LET is in 'Part $A^{\prime}$ and the interim suggestions of measures that might be used and studied are in 'Part B'

\begin{tabular}{|c|c|c|c|c|c|c|c|c|c|}
\hline \multirow[t]{2}{*}{ Domain } & \multirow[t]{2}{*}{ Measure } & \multirow[t]{2}{*}{ Decision } & \multirow{2}{*}{$\begin{array}{l}\text { Votes } \\
\text { Yes* }\end{array}$} & \multirow{2}{*}{$\frac{\text { Patient }}{\text { Agreed }}$} & \multicolumn{4}{|l|}{ Survey 2} & \multirow{2}{*}{$\begin{array}{l}\text { EMPRO } \\
\text { Score } \%\end{array}$} \\
\hline & & & & & In & Out & Unsure & Interim & \\
\hline \multirow[t]{4}{*}{ Disability } & PRTEE & $\sqrt{ }$ & $29(100)$ & $\sqrt{ }$ & $26(70.3)$ & $5(13.5)$ & $6(16)$ & $\mathrm{n} / \mathrm{a}$ & 57.0 \\
\hline & DASH & $x$ & & & $3(8.1)$ & $25(67.6)$ & $9(24.3)$ & $\mathrm{n} / \mathrm{a}$ & 66.9 \\
\hline & Quick DASH & $x$ & & & $22(59.5)$ & $9(24.3)$ & $6(16.2)$ & $\mathrm{n} / \mathrm{a}$ & 72.5 \\
\hline & Oxford Elbow Score & $x$ & & & $6(16.2)$ & $19(51.4)$ & $12(32.4)$ & $\mathrm{n} / \mathrm{a}$ & 66.6 \\
\hline \multicolumn{10}{|c|}{ Part B: interim suggestion for use in clinical trials and as a focus of future clinimetric research } \\
\hline \multirow[t]{2}{*}{ Domain } & Measure & Decision & Votes & Patient & Survey 2 & & & & EMPRO \\
\hline & & & Yes* & Agreed & In & Out & Unsure & Interim & Score $\%$ \\
\hline Function & PRTEE—relevant items & $\sqrt{ }$ & $30(96.8)$ & $\sqrt{ }$ & $24(64.9)$ & $5(13.5)$ & $8(21.6)$ & $33(89.2)$ & $\mathrm{n} / \mathrm{a}$ \\
\hline \multirow[t]{2}{*}{ Pain over specified time } & PRTEE pain subscale items 1,4 and $5 \dagger$ & $\sqrt{ }$ & $30(96.8)$ & & & & & & $\mathrm{n} / \mathrm{a}$ \\
\hline & Tennis Elbow Functional Scale & $x$ & $\mathrm{n} / \mathrm{a}$ & & $3(8.1)$ & $27(73)$ & $7(18.9)$ & $9(24.3)$ & 41.7 \\
\hline \multirow[t]{2}{*}{ Physical function capacity } & Pain-free grip strength & $\sqrt{ }$ & $26(86.7)$ & $\sqrt{ }$ & $15(40.5)$ & $11(29.7)$ & $11(29.7)$ & $24(64.9)$ & 32.9 \\
\hline & Maximum grip strength & $x$ & $\mathrm{n} / \mathrm{a}$ & & $6(16.2)$ & $17(46)$ & $14(37.8)$ & $12(32.4)$ & 25.1 \\
\hline \multirow[t]{3}{*}{ Pain on loading/activity } & Pain on gripping $\dagger$ & $\sqrt{ }$ & $25(83.3)$ & & $\mathrm{n} / \mathrm{a}$ & $\mathrm{n} / \mathrm{a}$ & $\mathrm{n} / \mathrm{a}$ & $\mathrm{n} / \mathrm{a}$ & $\mathrm{n} / \mathrm{a}$ \\
\hline & Tennis Elbow Functional Scale & $x$ & - & & $4(10.8)$ & $27(73)$ & $6(16.2)$ & $7(18.9)$ & 41.7 \\
\hline & PRTEE pain subscale items 2 and 3 & $x$ & $19(65.5)$ & $\sqrt{ }$ & $24(64.9)$ & $7(18.9)$ & $6(16.2)$ & $31(83.8)$ & $\mathrm{n} / \mathrm{a}$ \\
\hline \multirow[t]{2}{*}{ Participation } & Time off work & $\sqrt{ }$ & $22(73.3)$ & & $\mathrm{n} / \mathrm{a}$ & $\mathrm{n} / \mathrm{a}$ & $\mathrm{n} / \mathrm{a}$ & $26(70.3)$ & $\mathrm{n} / \mathrm{a}$ \\
\hline & Time off sport & $x$ & $18(60)$ & & $\mathrm{n} / \mathrm{a}$ & $\mathrm{n} / \mathrm{a}$ & $\mathrm{n} / \mathrm{a}$ & $21(56.8)$ & $\mathrm{n} / \mathrm{a}$ \\
\hline \multirow[t]{2}{*}{ QoL } & EQ5D & $x$ & $20(69)$ & & $\mathrm{n} / \mathrm{a}$ & $\mathrm{n} / \mathrm{a}$ & $\mathrm{n} / \mathrm{a}$ & $22(59.5)$ & $\mathrm{n} / \mathrm{a}$ \\
\hline & SF-12 & $x$ & $6(20.7)$ & & $\mathrm{n} / \mathrm{a}$ & $\mathrm{n} / \mathrm{a}$ & $\mathrm{n} / \mathrm{a}$ & $14(37.8)$ & $\mathrm{n} / \mathrm{a}$ \\
\hline \multirow[t]{3}{*}{ Participant Rating of Condition } & GROC & $x$ & $20(66.7)$ & $\sqrt{\ddagger}$ & $\mathrm{n} / \mathrm{a}$ & $\mathrm{n} / \mathrm{a}$ & $\mathrm{n} / \mathrm{a}$ & $21(56.8)$ & $\mathrm{n} / \mathrm{a}$ \\
\hline & Global Perceived Effect Score & $x$ & $\mathrm{n} / \mathrm{a}$ & & $\mathrm{n} / \mathrm{a}$ & $\mathrm{n} / \mathrm{a}$ & $\mathrm{n} / \mathrm{a}$ & $13(35.1)$ & $\mathrm{n} / \mathrm{a}$ \\
\hline & Patient Satisfaction Scale & $\mathrm{x}$ & $\mathrm{n} / \mathrm{a}$ & & $\mathrm{n} / \mathrm{a}$ & $\mathrm{n} / \mathrm{a}$ & $\mathrm{n} / \mathrm{a}$ & $17(46)$ & $\mathrm{n} / \mathrm{a}$ \\
\hline \multirow[t]{2}{*}{ Psychological } & Tampa Scale of Kinesiophobia & $x$ & $10(34.5)$ & $\sqrt{ } \ddagger$ & $\mathrm{n} / \mathrm{a}$ & $\mathrm{n} / \mathrm{a}$ & $\mathrm{n} / \mathrm{a}$ & $16(43.2)$ & $\mathrm{n} / \mathrm{a}$ \\
\hline & Hospital Anxiety and Depression Scale & $x$ & $\mathrm{n} / \mathrm{a}$ & & $\mathrm{n} / \mathrm{a}$ & $\mathrm{n} / \mathrm{a}$ & $\mathrm{n} / \mathrm{a}$ & $14(37.8)$ & $\mathrm{n} / \mathrm{a}$ \\
\hline
\end{tabular}

${ }^{*}$ Note that not all 31 attendees voted on all items (at least, 29 voted on 4 items) — due to time zone differences. See appendices for full data.

†Pain on gripping was voted in survey 1 , had no clinimetric evidence but was strongly supported for interim use - noting there were 2 (6.7\%) participants unsure.

¥Patients were asked for their opinions on which of the measures most closely measured their condition domain and was feasible clinically.

COS-LET, core outcome set for lateral elbow tendinopathy; DASH, Disabilities of the Arm, Shoulder and Hand Questionnaire; GROC, Global Rating of Change; PRTEE, Patient

Rated Tennis Elbow Evaluation; QoL, quality of life; SF-12, 12-item Short Form Health Survey.

note that the total EMPRO score was lower than other measures considered for inclusion. As previously identified, as the PRTEE was developed without patient involvement, its EMPRO score is, therefore, reduced as a consequence. ${ }^{7}$ However, the expert and patient groups within this COS development were concordant in their agreement that the PRTEE preferentially aligned with the disability domain. It was not possible to include measures in a COS-LET for the remaining domains, because there was either no instrument or a lack of instrument validation. In the interim, we decided on measures to recommend for validation studies and use in trials of LET.

The PRTEE function subscale was selected as an interim measure for the function domain, but concerns were raised that it mainly queries basic tasks and not higher-level tasks required in sports. This requires further investigation, along with the psychometric properties of the subscale. Patient responses from survey 1 favoured the Patient Specific Functional Scale for the function domain, but this was not supported by clinicians/ researchers. This scale can be tailored to athletic/high-level tasks so may be an area for future investigation.

The pain over a specified time domain had no suitable instruments following survey 2 . We resolved at the consensus meeting to recommend three questions from the PRTEE (pain at rest, least and worst over the last week) in the interim. The PRTEE had already been accepted for the COS-LET, thereby minimising patient burden-a key priority identified in the patient focus groups; however, the psychometric properties of these PRTEE items require further assessment.

Pain-free grip strength was recommended as an interim measure for physical function capacity domain, but it was not included in the final COS-LET due to limited validation in LET populations. With clear stakeholder approval, further validation work should be prioritised.

Measuring the pain on activity/loading domain was the source of a lengthy discussion, because the two options with sufficient psychometric evidence failed to reach consensus. Discussion then moved to rating pain on gripping as an interim measure. Gripping was raised as a common pain provoking activity in the patient focus groups. It had been voted in survey 1 to be in the COS-LET by patients and clinicians/researchers, but due to a lack of research it was not voted in survey 2. In the meeting, concerns were raised about standardising the gripping task-as this would be difficult across sexes and different work/sport contexts. We resolved to recommend using a Numerical Rating Scale to record pain during gripping in the interim and to prioritise its validation.

Time off work was recommended as an interim measure of the participation domain, but there were concerns regarding the definition of work and whether this was applicable to patients who were retired, unemployed, students or full-time parents/ 
carers. Due to the value of using time off work as part of health economic evaluation, however, it was agreed that it should still be used in the interim. It was recommended that future research should consider how this measure may be individualised to a patient's context.

No recommendations could be made for the domains of quality of life, patient rating of condition and psychology, primarily because there were no measures that had been validated for use in LET. For quality of life, the EQ5D narrowly missed interim selection and provoked mixed feelings from the patient focus groups. It was considered useful from a health economics perspective, for the calculation of quality adjusted life years. ${ }^{3031}$ Of the three instruments considered for the patient rating of condition domain, the Global Rating of Change (GROC) was preferred by the patient focus groups and is also regarded as an appropriate anchor for responsiveness analysis of other outcome measures. $^{32}{ }^{33}$ The Single Assessment Numerical Evaluation ${ }^{34}$ was proposed as an alternative option with the advantage that it can be used pretreatment and posttreatment, rather than relying on symptom recall, like the GROC. For the psychology domain, patients indicated the Tampa Scale of Kinesiophobia ${ }^{35}$ was more representative of their condition than anxiety and depression scales. Future studies should investigate the psychometric properties of these instruments for the LET population.

\section{Strengths and Limitations}

A strength of this study is that we included experts in LET (ie, patients and clinicians/researchers) from across the globe and followed a robust methodology that was published in advance.

There are several limitations that need to be considered in implementing the findings of this study. First, the COS-LET was developed on the basis of previously agreed on core domains for tendinopathy and are dependent on that work-any changes to those core domains will require revision of the COS-LET. Second, we were unable to recommend outcome measures for all of the core domains of tendinopathy-in which case we made interim recommendations. These interim recommendations should not be misconstrued as being part of the COS-LET, because they were made on the basis of opinions of participants without appropriate instrument validation. Third, we restricted the study to English outcome measures-using the COS-LET in non-English language situations requires validation. Fourth, we did not include a patient in our steering committee.

\section{Areas for future research}

Future research is required to establish valid and feasible measures across all health-related tendinopathy domains in patients who have LET. We have identified some targets herein: PRTEE subscales/items, pain on gripping, GROC, EQ5D and Tampa Scale of Kinesiophobia.

\section{CONCLUSION}

The PRTEE should be used in all future studies related to LETespecially for the disability domain. Time off work, pain-free grip strength and a Numerical Rating Scale measuring pain on gripping should also be used until future studies recommend alternative, more robust, measures of participation in life activities, physical function capacity and pain on activity/loading. A COS-LET Tool, containing these recommended measures, has been composed (see online supplemental file 4). Further work is required to (a) validate the interim measures for use in research involving persons with LET and (b) develop/validate suitable measures of the patient rating of condition, quality of life and psychological factors domains.

\section{Key messages}

What is already known?

- Core outcome sets (COSs) are recommended for research and clinical practice to facilitate comparison and meta-analysis of results.

- COSs for tendinopathies should map to the established list of nine core health-related domains.

- Lateral elbow tendinopathy (LET) is a common clinical problem that has received considerable research attention.

- There is no agreed COS-LET_limiting meta-analysis.

What are the findings?

- The COS-LET consists of the Patient Rated Tennis Elbow Evaluation - it should be used to capture the disability domain in clinical settings and in all research of this condition.

- The Patient Rated Tennis Elbow Evaluation and its subscales offer insights into the domains of pain and function in addition to disability.

- When measuring participation, physical function capacity and pain on loading, we recommend as interim measures, respectively, time off work, pain-free grip strength and a numerical rating scale for pain on gripping.

- Further work is required to validate many of the measures used in clinical practice.

How might it impact on clinical practice in the future?

- Systematic use of the COS-LET will allow for meta-analysis of research studies and comparison of results between different clinical practices during service evaluation.

- Meta-analysis and network meta-analysis across multiple research trials that apply the COS-LET will increase understanding of treatment effectiveness for people with LET.

\section{Author affiliations}

${ }^{1}$ Orthopaedic Department, University Hospitals of Derby and Burton NHS Foundation Trust, Derby, UK

${ }^{2}$ Princess Elizabeth Orthopaedic Centre, Royal Devon and Exeter Hospital, Exeter, UK ${ }^{3}$ Health Services and Policy Research Group, University of Exeter Medical School, Exeter, UK

${ }^{4}$ School of Health \& Rehabilitation Sciences: Physiotherapy, University of Queensland, Brisbane, Queensland, Australia

${ }^{5}$ Orthopaedic Department, Sheffield Teaching Hospitals NHS Foundation Trust, Sheffield, UK

${ }^{6}$ Upper Limb Unit, Wrightington Hospital, Wrightington, UK

${ }^{7}$ Department of Trauma and Orthopaedics, University Hospitals Sussex NHS

Foundation Trust, Brighton, UK

${ }^{8}$ Brighton and Sussex Medical School, Brighton, UK

${ }^{9}$ Menzies Health Institute Queensland, Griffith University, Gold Coast, Queensland, Australia

${ }^{10}$ School of Health Sciences \& Social Work, Griffith University, Gold Coast, Queensland, Australia

Twitter Marcus Bateman @MarcusBatemanPT, Jonathan P Evans @jon_evans_ uk, Viana Vuvan @vianavuvan, Val Jones@Valjones2305val, Adam C Watts @myelbowdoc, Leanne M Bisset @leannebisset and Bill Vicenzino @Bill_Vicenzino

Acknowledgements The steering committee would like to acknowledge the contributions of the core outcome set for lateral elbow tendinopathy participant group, who donated their time voluntarily. Without them, this project would not have been possible.

Collaborators COS-LET consensus group: Isabel Andia (BioCruces Health Research Institute, Spain), Paolo Arrigoni (Università degli Studi di Milano, Italy), Canan Aydin (Metin Sabanci Baltalimani Bone Diseases Training and Research Hospital, Turkey), Paul Barratt (Salford Royal Foundation Trust, UK), Ram Chidambaram (MGM Healthcare, India), Joshua Cleland (School of Medicine, Tufts University, Medford, 
Massachusetts, USA), Brooke Coombes (Griffith University, Australia), Uri Farkash (Assuta Ashdod University Hospital, Israel), Taco Gosens (Elisabeth Tweesteden Hospital, the Netherlands), Luke Heales (Central Queensland University, Australia), Martin Kroslak (University of New South Wales, Australia), Chris Littlewood (Manchester Metropolitan University, UK), Joy MacDermid (University of Western Ontario, Canada), George Murrell (University of New South Wales, Australia), Morten Olaussen (University of Oslo, Oslo, Norway), John Orchard (University of Sydney, Australia), Tuomo Pienimaki (Oulu Regional Institute of Occupational Health, Finland), Amar Rangan (James Cook University Hospital, UK), Eirik Solheim (University of Bergen, Norway), Dimitrios Stasinopoulos (University of West Attica, Greece), Jill Thomas (Wrightington, Wigan and Leigh NHS Foundation Trust, UK), Timothy Tyler (Lenox Hill Hospital, New York City, New York, USA), Danielle Van Der Windt (Keele University, UK), Roger Van Riet (Monica Hospital Antwerp, Belgium), Allan Wang (St John of God Subiaco Hospital, Australia), Esther Waugh (University of Toronto, Canada), Jennifer Moriatis Wolf (University of Chicago, Chicago, Illinois, USA), Tony Wright (Curtin University, Australia), Eva Zeisig (Umeå University, Sweden), Ming Hao Zheng (University of Western Australia, Australia), CH (patient representative, UK), Priyesh Mistry (patient representative, UK), RN (patient representative, UK), Ruth Newsome (patient representative, UK), MW (patient representative, UK), Mark Ford (patient representative, Australia), Karen Jackson (patient representative, Australia), Phuong Le (patient representative, Australia) and Joo Yeun Song (patient representative, Australia).

Contributors MB and JPE share joint first authorship and were responsible for study concept, design and the writing of this manuscript. BV was responsible for study concept, design, oversight and the writing of this manuscript. AW, JP, VJ, VV and LMB were responsible for study concept, design and the editing of this manuscript. All authors contributed to the conduct of the study and have approved the final version of the manuscript. MB is guarantor.

Funding This work is not funded and relies on the individuals involved donating their time. Open access publication fees were funded by the University Hospitals of Derby and Burton NHS Foundation Trust Charity.

Competing interests $\mathrm{MB}$ and JPE contributed equally and share first authorship. $M B, J P E, A W, J P, V J, V V, L M B$ and BV declare that they have no competing interests. The core outcome set for lateral elbow tendinopathy (COS-LET) consensus group has no competing interests except for Joy MacDermid, who is the author of the Patient Rated Tennis Elbow Evaluation (PRTEE). Her responses were removed from survey and consensus voting related to the use of the PRTEE in the COS-LET.

\section{Patient consent for publication Not applicable.}

Ethics approval This study involves human participants and was approved by the University of Queensland's research ethics committee (reference number: 2020001340). Participants gave informed consent to participate in the study before taking part.

Provenance and peer review Not commissioned; externally peer reviewed.

Data availability statement All data relevant to the study are included in the article or uploaded as supplementary information. Not applicable.

Supplemental material This content has been supplied by the author(s). It has not been vetted by BMJ Publishing Group Limited (BMJ) and may not have been peer-reviewed. Any opinions or recommendations discussed are solely those of the author(s) and are not endorsed by BMJ. BMJ disclaims all liability and responsibility arising from any reliance placed on the content. Where the content includes any translated material, BMJ does not warrant the accuracy and reliability of the translations (including but not limited to local regulations, clinical guidelines, terminology, drug names and drug dosages), and is not responsible for any error and/or omissions arising from translation and adaptation or otherwise.

Open access This is an open access article distributed in accordance with the Creative Commons Attribution 4.0 Unported (CC BY 4.0) license, which permits others to copy, redistribute, remix, transform and build upon this work for any purpose, provided the original work is properly cited, a link to the licence is given, and indication of whether changes were made. See: https://creativecommons.org/ licenses/by/4.01.

\section{ORCID iDs}

Marcus Bateman http://orcid.org/0000-0002-3203-506X

Jonathan P Evans http://orcid.org/0000-0003-0090-1012

Bill Vicenzino http://orcid.org/0000-0003-0253-5933

\section{REFERENCES}

1 Walker-Bone K, Palmer KT, Reading I, et al. Prevalence and impact of musculoskeletal disorders of the upper limb in the general population. Arthritis Rheum 2004;51:642-51.

2 Scott A, Squier K, Alfredson H, et al. Icon 2019: international scientific tendinopathy symposium consensus: clinical terminology. Br J Sports Med 2020;54:bjsports2019-100885.
3 Evans JP, Smith CD, Fine NF, et al. Clinical rating systems in elbow research-a systematic review exploring trends and distributions of use. J Shoulder Elbow Surg 2018;27:e98-106.

4 Vicenzino B, de Vos R-J, Alfredson H. Icon 2019_-International scientific tendinopathy symposium consensus: there are nine core health-related domains for tendinopathy (core domains): Delphi study of healthcare professionals and patients. Br J Sports Med 2019;54.

5 Reeve BB, Wyrwich $\mathrm{KW}$, Wu AW, et al. ISOQOL recommends minimum standards for patient-reported outcome measures used in patient-centered outcomes and comparative effectiveness research. Qual Life Res 2013;22:1889-905.

6 Prinsen CAC, Vohra S, Rose MR, et al. How to select outcome measurement instruments for outcomes included in a "Core Outcome Set" - a practical guideline. Trials 2016:17:449.

7 Evans JP, Porter I, Gangannagaripalli JB, et al. Assessing patient-centred outcomes in lateral elbow tendinopathy: a systematic review and standardised comparison of English language clinical rating systems. Sports Med Open 2019;5:10.

8 Boers M, Kirwan J, Tugwell P. OMERACT Handbook, 2018.

9 Valderas JM, Ferrer M, Mendívil J, et al. Development of EMPRO: a tool for the standardized assessment of patient-reported outcome measures. Value Health 2008;11:700-8.

10 Kirkham JJ, Gorst S, Altman DG, et al. Core outcome Set-STAndards for reporting: the COS-STAR statement. PLoS Med 2016:13:e1002148.

11 Expertscape Inc. Expertscape. USA 2000-2020, 2000.

12 Elsevier BV. Scopus ${ }^{\circledR}$ Expertly curated abstract \& citation database, 2020. Available: https://www.elsevier.com/en-in/solutions/scopus

13 Ouzzani M, Hammady H, Fedorowicz Z, et al. Rayyan-a web and mobile APP for systematic reviews. Syst Rev 2016;5:210.

14 Macdermid J. The Patient-Rated Tennis Elbow Evaluation (PRTEE)@ User Manual 2008.

15 Vincent J, MacDermid JC. Patient-rated tennis elbow evaluation questionnaire. J Physiother 2014;60:240

16 Shafiee E, MacDermid JC, Walton D, et al. Psychometric properties and cross-cultural adaptation of the Patient-Rated tennis elbow evaluation (PRTEE); a systematic review and meta-analysis. Disabil Rehabil 2021;24:1-16.

17 Kennedy C, Beaton D, Solway S. The DASH and Quick DASH Outcome Measure User's Manual. 3 edn. Toronto, Ontario: Institute for Work \& Health, 2011.

18 Stratford Pet al. Assessing disability and change on individual patients: a report of a patient specific measure. Physiotherapy Canada 1995;47:258-63.

19 Lowe KA. The test retest reliability, construct validity, and responsiveness of the tennis elbow function scale. University of Alberta, 1999.

20 Hudak PL, Amadio PC, Bombardier C. Development of an upper extremity outcome measure: the DASH (disabilities of the arm, shoulder and hand) [corrected]. The Upper Extremity Collaborative Group (UECG). Am J Ind Med 1996;29:602-8.

21 Gummesson C, Atroshi I, Ekdahl C. The disabilities of the arm, shoulder and hand (DASH) outcome questionnaire: longitudinal construct validity and measuring selfrated health change after surgery. BMC Musculoskelet Disord 2003:4:11.

22 Janssen S, De Smet L. Responsiveness of the DASH questionnaire for surgically treated tennis elbow. Acta Chir Belg 2008;108:583-5.

23 Beaton DE, Wright JG, Katz JN, et al. Development of the QuickDASH: comparison of three item-reduction approaches. J Bone Joint Surg Am 2005:87:1038-46.

24 Polson K, Reid D, McNair PJ, et al. Responsiveness, minimal importance difference and minimal detectable change scores of the shortened disability arm shoulder hand (QuickDASH) questionnaire. Man Ther 2010;15:404-7.

25 Dawson J, Doll H, Boller I, et al. The development and validation of a patientreported questionnaire to assess outcomes of elbow surgery. J Bone Joint Surg Br 2008;90:466-73

26 Dawson J, Doll H, Boller l, et al. Comparative responsiveness and minimal change for the Oxford elbow score following surgery. Qual Life Res 2008;17:1257-67.

27 Stratford PW, Levy DR. Assessing valid change over time in patients with lateral epicondylitis at the elbow. Clinical Journal of Sport Medicine 1994;4:88-91.

28 Smidt N, van der Windt DA, Assendelft WJ, et al. Interobserver reproducibility of the assessment of severity of complaints, grip strength, and pressure pain threshold in patients with lateral epicondylitis. Arch Phys Med Rehabil 2002;83:1145-50.

29 Lim ECW. Pain free grip strength test. J Physiother 2013;59:59.

30 EuroQol Group. EuroQol--a new facility for the measurement of health-related quality of life. Health Policy 1990;16:199-208.

31 Connelly LB. Economic Evaluations with Pre-Scored Health Status Instruments. In: Oakland T, Mpofu E, eds. Rehabilitation and health assessment: applying ICF guidelines. New York: Springer, 2010: 163-88.

32 Kamper S. Global rating of change scales. Aust J Physiother 2009:55:289.

33 Kamper SJ, Maher CG, Mackay G. Global rating of change scales: a review of strengths and weaknesses and considerations for design. J Man Manip Ther 2009;17:163-70.

34 Furtado R, MacDermid J. Clinimetrics: single assessment numeric evaluation. J Physiother 2019;65:111.

35 Swinkels-Meewisse EJCM, Swinkels RAHM, Verbeek ALM, et al. Psychometric properties of the Tampa scale for kinesiophobia and the fear-avoidance beliefs questionnaire in acute low back pain. Man Ther 2003;8:29-36. 